\title{
Research on Dynamic Braking Torque Test Method of Brake Motor
}

\author{
Zheng-qiu Huang ${ }^{1, *}$, Guo-feng $\mathrm{Li}^{2}$, Yao-ting Tong ${ }^{1}$, Wei-ping Ouyang ${ }^{1}$ \\ ${ }^{1}$ Shanghai Institute of Special Equipment Inspection and Technical Research, 200062, Shanghai \\ ${ }^{2}$ Shanghai Fengxian District Special Equipment Inspection Institute, 201406, Shanghai
}

\begin{abstract}
Through the brief introduction of the working principle of the brake motor, combined with the theoretical analysis of the brake motor dynamic brake torque test method (directtest method and indirect test method), it is concluded that the selection of the test method is closely related. According to the sensor type, installation position, test range and accuracy requirements. The differences between direct measurement method and indirect measurement method are compared, their respective measurement methods are introduced,and their advantages and disadvantages are summarized. According to the test requirements, the appropriate test method can be selected, and the direct test method and indirect test method can be selected at the same time,to ensure the safety and stability of product
\end{abstract}

\section{Introduction}

Braking motor is a kind of motor with braking function,which has the characteristics of integrating drive and braking. It has compact structure and accurate positioning. According to the structure type, it can be divided into conical rotor braking motor, side magnetic brake motor, disc brake motor, DC electromagnetic braking motor, braking motor with AC brake and so on[1][2]. Braking motor has the working characteristics of intermittent movement, often starting and braking, which mainly plays the role of starting operation and stopping braking in various crane mechanisms, and its application has also expanded from the original lifting machinery industry to textile, machine tool, food and other machinery industries. Since the first cone-shaped rotor brake motor came out in 1920s, after long-term development, it has formed a situation of coexistence of various types of brake motor, such as side magnet brake, disc brake and belt brake. With the rapid growth of brake motor varieties and quantity, the inspection and quality supervision of related products has become an important topic. TSG.Q7002-2019[3] "regulation for type test of lifting appliance" issued by the State Administration of market supervision clearly stipulates the type test items and requirements of brake motor.

The braking performance of braking motor is directly related to the safety and reliability of lifting machinery, and its main indexes are static braking torque and dynamic braking torque[4]. At present, there are few test devices for brake motor products at home and abroad[5], German Demag Crane Co., Ltd. has established a special crane brake test system ,Although Jiaozuo brake Co., Ltd. and Jiangxi Huawu brake Co., Ltd. have special brake test system and quality control, due to the limitations of capital and technology, the test equipment and detection methods used by enterprises are relatively backward, and generally only static brake torque test can be used. It can be carried out in the braking performance test, and the dynamic braking torque test ability of the device is very few, the test ability is limited. generally only static brake torque test can be done in brake performance test, and there are few devices with dynamic brake torque test ability, but the static braking torque can not effectively reflect the braking performance of the braking motor under dynamic load, and the dynamic braking torque can truly reflect the braking performance of the braking motor under the actual working conditions, which has more important significance.

In addition, with the development of crane industry, more accurate and larger measuring range test bench is needed to meet the existing shortcomings. At the same time, in the brake working principle research, product stability inspection, safety accident analysis and other fields also have the role of this crew. In the process of new product development, the brake test bench can also be used to simulate the actual working conditions, and the performance parameters in the actual work can be obtained, so as to ensure that the new product can complete the work task safely and stably after it is put on the market.

\section{Working principle of brake motor}

The brake motor drives the load to run through the transmission system, the power supply is disconnected when running to the stop, the braking motor brakes, and at the same time produces braking torque. After a period of time, the braking motor turns to a certain angle and slows down to the stop state.The energy conversion in

\footnotetext{
* Corresponding author: huangzq@ssei.cn
} 
the braking process is as follows: when the motor starts and runs, the motor converts the electric energy into mechanical energy; when braking stops, the braking system absorbs the mechanical energy and converts it into heat energy by friction braking,and then stores and emits it. According to the law of energy conservation, the mechanical energy obtained by the motor driving the load in the braking process is equal to the energy absorbed by the braking motor.

For example, when the crane using the brake motor as the lifting mechanism stops running, the energy absorbed by the brake motor is:

$$
Q=T_{z} q_{z}=\frac{1}{2} m v^{2}+m g h+\frac{1}{2} J W^{2}
$$

In the formula:

$T_{z}$-Braking torque;

$q_{z}$-Brake rotation angle ;

$m$-Weight of load ;

$v$-Initial braking speed of lifting objects ;

$h$ - Braking distance ;

$J$-Moment of inertia;
$W$-angular velocity.

\section{Test method}

There are many methods to test the dynamic braking torque of braking motor. All kinds of test methods have large or small errors in the measurement. Generally speaking, there are mainly direct test method and indirect test method[6][7] .

\subsection{Direct test method}

The direct test method is to measure the dynamic braking torque directly by the torque sensor. Generally, the torque sensor is connected in series to the transmission shaft between the brake motor and the flywheel group under test[8]. As shown in Fig. 1, the braking torque measured by this method is the instantaneous value of the dynamic change with time.

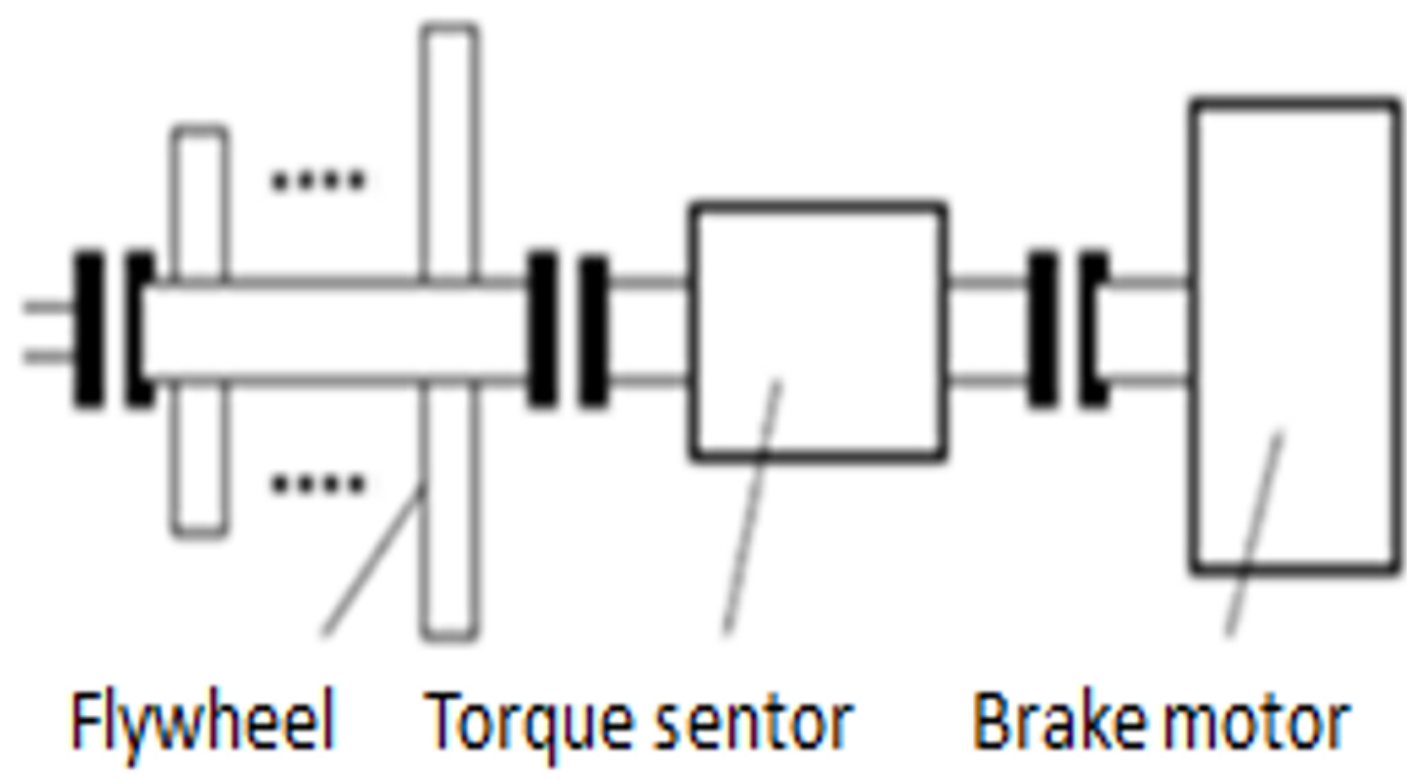

Fig. 1. schematic diagram of direct measurement

Because the torque sensor is installed between the brake motor and the flywheel group, in theory,the torque sensor actually measures the difference between the total flywheel torque $G D^{2}$ (Including brake motor, flywheel group, transmission shaft) and the flywheel torque $G D_{1}^{2}$ (flywheel torque between brake motor and torque sensor).

But the transmission shaft and bearing seat between braking motor and torque sensor will have a certain efficiency loss in energy transfer, coupled with the influence of resistance torque, the actual measured torque value will be smaller than the real value.In order to obtain a more accurate braking torque value, the measured value can be multiplied by a correction factor 1 to correct the measurement results, the formula is as follows:

$$
I=\frac{G D^{2}}{G D^{2}-G D_{1}^{2}}
$$

From the above analysis, it can be seen that the test method ignores the influence of energy loss and friction torque between the measured braking motor and the torque sensor in the braking stage, and the requirements 
for the measurement range and accuracy of the torque sensor are also high.

\subsection{Indirect test method}

The so-called indirect measurement method is not directly through the test of the brake force or torque (that is, not using force or torque sensor), but through the test of other parameters in the system [9][10], such as angular velocity or angular displacement, The braking torque value can be obtained directly through some theoretical formula derivation and calculation. There are generally the following indirect test methods:

\subsubsection{Measuring the change of angular speed}

By measuring the change of angular speed $w_{z}$ during braking, The insantaneous value of torque is calculated. The formula is as follows:

$$
T_{z}=J_{z} \dot{W}_{z}
$$

In the formula :

$T_{z}$-Braking torque;

$J_{z}$-Moment of inertia of Inertial Flywheel system;

$W_{z}$-The braking initial angular speed of inertial flywheel system.

\subsubsection{Measuring the angle of rotation}

By measuring the angle of rotation during braking $\theta \mathrm{z}$, Recording simulated moment of inertia Jz and the initial angular speed of brakingwz, The average braking torque is calculated and the formula is as follows:

$$
T_{z}=\frac{1}{2} J_{z} w_{z}^{2} / q_{z}
$$

In the formula :

$q_{z}$-Brake angle.

\subsubsection{Measuring flywheel torque, rotating speed and braking time}

Though the parameters such as flywheel torque, rotating speed and braking time, the average torque is calculated, and the calculationg formula is as follows:

$$
T_{z}=\frac{\sum_{i=0}^{n} G D_{i}^{2}+G D_{0}^{2}}{375} \times \frac{n_{0}}{t}-M_{f}
$$

In the formula :

$G D_{i}^{2}$-Sec. i Flywheel torque of flywheel;

$G D_{0}^{2}$-Flywheel torque inherent in the system;

$n_{0}$-Initial braking speed;

$M_{f}$-Friction torque.

This method is tedious, the friction torque should be tested in the early stage, and there is a lot of preparatory work, and the calculated value is an average value.

\subsubsection{Through rotating speed encoder}

Through continuous acquisition of rotating speed encoder at equal time intervals, the speed values at different times are obtained $n_{0} 、 n_{1}, n_{2}, \ldots n_{i-1}$,

$n_{i}$.The braking torque at different times is calculated, and the formula is as follows :

$$
\begin{gathered}
T_{z 1}=\frac{\left(n_{0}-n_{1}\right) J_{z}}{9.55 t} h \\
T_{z 2}=\frac{\left(n_{1}-n_{2}\right) J_{z}}{9.55 t} h \\
\cdots \cdots \\
T_{z i}=\frac{\left(n_{i-1}-n_{i}\right) J_{z}}{9.55 t} h
\end{gathered}
$$

In the formula :

$T_{Z i}$-Sec. i Segment average braking torque;

$n_{i}$-Sec. $i$ Period of time at the end of the speed;

$h$-Mechanical efficiency of transmission system.

To sum up, the indirect test method is the braking torque value obtained through indirect test, without considering the influence of other factors in the real braking. When the torque or force sensor is affected by the range and other factors, it can be used to correct and check, compare and calculate, and finally get a more accurate braking torque.

\section{Analysis of test method}

Through the above analysis, according to the different use of the brake motor under test, the following conclusions can be drawn:

1 , The direct measurement method is limited by the range of the sensor, and the suitable sensor needs to be replaced according to the measured sample in the testing process, but the indirect measurement method is basically not limited by this factor.

2, For the direct measurement method, because there are stereotyped sensors and instruments on the market, it is more convenient to build a test system.

3, For the indirect measurement method, the measured data is a piecewise average value, while the direct measurement method can obtain the instantaneous value. The braking torque $(\mathrm{T})$ is a function $\mathrm{T}(\mathrm{t})$ that changes with time. The dynamic curve of the braking torque can be obtained by the simulation of the advance data analysis software, which can reflect the dynamic characteristics of some parameters[11].

4 , For general brake motor manufacturers or product quality testing center, the test purpose is clear. It mainly tests whether the dynamic braking torque of brake motor can ensure the safety and reliability of braking process. Therefore, some simple and easy test methods can be adopted, such as directly measuring the brake torque by using torque sensor. 
5, For the manufacturers, scientific research institutions or universities that need to carry out the research and development of brake motor products, the measurement range and accuracy of the parameters in the braking process are required to be higher. At the same time, the real-time acquisition and analysis of the test data are also required. The direct test method and indirect test method can be used together, and the relevant measurement parameters can be compared and analyzed.

\section{Conclusion}

Through the introduction of the development process and application occasions of the brake motor, as well as the brief introduction of the working principle of the brake motor, combined with the theoretical analysis of the dynamic brake torque test method of the brake motor, it is concluded that the selection of the test method is closely related to the sensor type, installation position, test range and accuracy requirements. The differences between direct measurement method and indirect measurement method are compared, and their advantages and disadvantages are summarized. According to the requirements of test, the appropriate test method can be selected, and the direct test and indirect test method can be selected at the same time.

Because there are some shortcomings in the test methods, whether direct measurement method or indirect measurement method, in the future research, we can consider combining two kinds of prevention, comparing and correcting the data measured by the two methods, in order to obtain higher accuracy and more reliable measurement data.To ensure the safety and stability of product

\section{Acknowledgements}

I would like to express my sincere thanks to Shanghai Institute of special equipment supervision and inspection technology and Shanghai Fengxian special equipment supervision and Inspection Institute. With the help of my colleagues, I completed this paper.

Last my thanks would go to my beloved family for their loving considerations and great confidence in me all through these years. I also owe my sincere gratitude to my friends who gave me their help and time in listening to me and helping me work out my problems during the difficult course of the thesis.

\section{References}

1. Specification for three-phase Asynchronous braking of conical rotor Brake, JB/T 7562-2002 YEZX Series, Mechanical Industry Standard of the people's Republic of China [S].2002.2002.

2. Specification for JB/T 7563-2005 YZE Series electromagnetic Brake three-phase Asynchronous Motor for Mechanical Industry Standard of the people's Republic of China [S]. 2005.
3. Special equipment safety technical specification, TSG.Q7002-2019 Regulation for Type Test of Lifting Appliances[S].2019.

4. Wang Quanwei.research on inertia test system of crane brake[D].Taiyuan University of Science and Technology,2008.

5. Liu Wu Sheng, Lu Jian Chao. Present situation and development trend of brake at home and abroad [J]. Port handling, 2000 (add): 22-30.

6. Bai Jianyi, Su Wensheng, Li Yunfei, and so on. Design of dynamic braking torque Test device for Brake Motor [J]. Lifting and Transportation Machinery, 2014 (4): 12-15.

7. Lu Zehui. Research and development of crane brake experimental system [D]. Wuhan University of technology, 2011.

8. Lu Jianhu, Zhao Menglin. Industrial brake inertia test bed $[\mathrm{J}]$. Lifting and transportation machinery 2008 (6): 139-141.

9. Gu Xupo, Su Wensheng, hundred perseverance, etc. Dynamic braking torque test of crane brake based on deceleration method [J]. Lifting and Transportation Machinery, 2014 (6): 84-87.

10. Huang manguo, Tao Yuanfang. Application of sensor in signal acquisition system of brake test bed [J], micro nano electronic technology, 2007 (7 / 8): 183-184.

11. Mei ShunQI, Zhang Zhiming. Principle of PCI-1710 data acquisition and its application in industry [J]. Modern manufacturing engineering, 2006 (7): 31-32 